\title{
Factores que condicionan severidad de colecistitis grado I vs. grado II en mujeres adultas
}

\author{
Factors associated with severity of grade I cholecystitis \\ vs grade II in adult female patients \\ Juan de Dios Díaz-Rosales, ${ }^{*}$ Oscar I Ortiz-Ruvalcaba, \\ Gilberto Mena-Arias, ${ }^{*}$ Sergio Morales-Polanco*
}

Palabras clave: Colecistitis, colecistectomía, cálculos biliares, mujeres.

Keywords:

Cholecystitis, cholecystectomy, gallstones, women.

* Cirugía General y Endoscopia Gastrointestinal, Hospital General de Zona No. 35, Instituto Mexicano del Seguro Social.

* Gastroenterología y Endoscopia Gastrointestinal, Hospital General de Zona No. 35, Instituto Mexicano del Seguro Social, Departamento de Ciencias Médicas, Universidad Autónoma de Ciudad Juárez.

Recibido: 28/07/2019 Aceptado: 04/11/2019

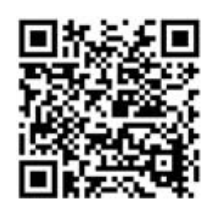

\section{RESUMEN}

Introducción: La colelitiasis es un problema de salud pública en México. La obesidad se considera un factor de riesgo de la génesis de esta enfermedad; sin embargo, no está bien definido su papel con respecto a la influencia en la severidad del cuadro de colecistitis. Objetivo: Evaluar los factores que condicionan la severidad en un cuadro de colecistitis (grado I vs. grado II, según las guías de Tokio 18) en mujeres en un hospital público en Ciudad Juárez. Material y métodos: Estudio transversal en mujeres con colecistitis grado I y II sometidas a colecistectomía laparoscópica. Se evaluaron y compararon las siguientes variables: edad, peso, talla, índice de masa corporal (IMC), cintura, cadera, índice cintura/cadera (ICC), tensión arterial, glucosa, colesterol, lipoproteínas de alta densidad (HDL), triglicéridos, leucocitos, neutrófilos, tiempo quirúrgico, presencia de diabetes tipo 2 (DM2), hipertensión arterial (HTA), hipertrigliceridemia, hipercolesterolemia, dislipidemia, obesidad abdominal, obesidad por ICC y síndrome metabólico (SM). Resultados: Se estudiaron 132 pacientes, sólo se observó diferencia estadísticamente significativa y significancia clínica en el promedio de triglicéridos ( 155.9 vs. $178.4 ; p=0.008)$, y en la presencia de hipertrigliceridemia $(40.5 \%$ vs. $70.8 \%$; $=0.001)$. Conclusiones: La obesidad medida por el IMC, cintura y/o ICC no parecen tener relación con el grado de severidad de la colecistitis (grado I vs. grado II), mientras que el promedio de triglicéridos y la presencia de hipertrigliceridemia sí pueden actuar como factores agravantes de la colecistitis aguda grado II.

\section{ABSTRACT}

Introduction: Cholelithiasis is a great public health problem in Mexico. Obesity plays a role during genesis of gallbladder disease, however, is not clear if it affects the severity of cholecystitis. Objective: The aim of this study was to evaluate risk factors for severity in cholecystitis (grade I vs grade II) in female patients. Material and methods: Cross-sectional study to evaluate risk factors to severity during cholecystitis (grade I vs grade II) in women that were underwent to laparoscopic cholecystectomy. We evaluated and compared: age, weight, height, body mass index (BMI), waist, hip, waist-hip index (WHI), blood pressure, glucose, cholesterol, high density lipoproteins (HDL), triglycerides, leucocytes, neutrophils, surgical time, presence of type 2 diabetes, arterial hypertension, hypertriglyceridemia, hypercholesterolemia, dyslipidemia, central obesity, obesity by WHI, and metabolic syndrome. Results: We analyzed 132 patients that were underwent to laparoscopic cholecystectomy. Only triglycerides (155.9 vs 178.4; $p=0.008$ ) and presence of hypertrygliceridemia $(40.5 \%$ vs $70.8 \%$; $p=$ $0.001)$ were statistical different. Conclusions: Neither obesity by BMI nor central obesity (by waist or WHI) have a relation with severity of cholecystitis, but levels of triglycerides and presence of hypertriglyceridemia could be risk factor for severity (grade II).

Citar como: Díaz-Rosales JD, Ortiz-Ruvalcaba OI, Mena-Arias G, Morales-Polanco S. Factores que condicionan severidad de colecistitis grado I vs. grado II en mujeres adultas. Cir Gen. 2020; 42(1): 6-12. doi: 10.35366/92705 


\section{INTRODUCCIÓN}

L a colelitiasis es un problema de salud pública en la actualidad. ${ }^{1}$ Estudios en autopsias revelan que $12 \%$ de los hombres y $24 \%$ de las mujeres sufren de esta entidad. ${ }^{2}$ Aunque $80 \%$ de los pacientes con colelitiasis pueden permanecer asintomáticos, el resto presenta síntomas como el cólico biliar, y complicaciones como colecistitis, coledocolitiasis, pancreatitis y colangitis, entre otras. ${ }^{2}$

Los factores de riesgo de colelitiasis y colecistitis están bien definidos. Mientras que la obesidad se considera clásicamente como un factor etiológico, se sugiere que la obesidad central y el índice de cintura/cadera (ICC) desempeñan un papel importante en la severidad de la colecistitis. ${ }^{3}$

El objetivo de este estudio es evaluar qué factores pueden condicionar la aparición de un cuadro de colecistitis grado II en una población femenina sometida a colecistectomía laparoscópica.

\section{MATERIAL Y MÉTODOS}

Se realizó un estudio transversal de enero a diciembre de 2018 en el Hospital General

Tabla 1: Criterios diagnósticos de colecistitis aguda según las guías Tokio 18.

A. Signos locales de inflamación

1. Signo de Murphy

2. Dolor/masa/sensibilidad en el CSD

B. Signos de inflamación sistémica

1. Fiebre

2. Elevación de PCR

3. Elevación de leucocitos

C. Hallazgos en imagen

Hallazgos característicos de colecistitis aguda* Sospecha diagnóstica:

un elemento de $\mathrm{A}+$ un elemento de $\mathrm{B}$

Diagnóstico definitivo:

un elemento de $\mathrm{A}+$ un elemento de $\mathrm{B}+\mathrm{C}$

$\mathrm{CSD}=$ cuadrante superior derecho; $\mathrm{PCR}=$ proteína

$\mathrm{C}$ reactiva.

* Hallazgos de imagen: engrosamiento de la pared vesicular, líquido perivesicular, signo sonográfico de Murphy positivo. de Zona No. 35 del Instituto Mexicano del Seguro Social (HGZ No. 35, IMSS) en Ciudad Juárez, México. Se estudiaron pacientes femeninos de entre 18 y 65 años, ingresadas a hospital con diagnóstico de colecistitis aguda y que fueron sometidas a colecistectomía laparoscópica.

Se excluyeron las pacientes con enfermedades hepáticas como cirrosis, quistes o tumores hepáticos de cualquier etiología, cáncer del resto del aparato digestivo, embarazo y pacientes cuya colecistectomía hubiera sido convertida de laparoscópica a abierta.

Se dividieron las pacientes en dos grupos, grupo 1 con pacientes con colecistitis aguda sin otra complicación o colecistitis grado I y grupo 2 con pacientes con colecistitis aguda complicada (coledocolitiasis, colangitis, pancreatitis biliar, mucocele, empiema, etc.) sin disfunción orgánica o grado II. El diagnóstico de colecistitis aguda, así como la diferenciación de colecistitis aguda grado I y grado II se determinó con base en los hallazgos operatorios y los criterios de las guías Tokio 18 (Tablas 1 y 2). ${ }^{4}$

Se llevó a cabo la colecistectomía laparoscópica como tratamiento del cuadro agudo. La operación fue realizada por el mismo equipo quirúrgico usando la técnica estándar de cuatro puertos. ${ }^{5}$ El procedimiento se efectuó durante el mismo internamiento.

Se midieron las siguientes variables: edad, peso, talla, índice de masa corporal (IMC), cintura, cadera, índice cintura/cadera (ICC), tensión arterial, glucosa, colesterol, lipoproteínas de alta densidad (HDL), triglicéridos, leucocitos, neutrófilos, tiempo quirúrgico, presencia de diabetes tipo 2 (DM2), hipertensión arterial (HTA), hipertrigliceridemia $(\geq$ $150 \mathrm{mg} / \mathrm{dl}$ ), hipercolesterolemia ( $\geq 200 \mathrm{mg} /$ dl), dislipidemia (alteración del colesterol y/o triglicéridos), obesidad abdominal (cadera 88 $\mathrm{cm})$, obesidad por ICC ( $\geq 0.85)$ y síndrome metabólico (SM).

El peso y la talla fueron medidos con la paciente descalza y con bata, el IMC fue calculado dividiendo el peso $(\mathrm{kg})$ entre la altura al cuadrado (metros). La circunferencia de cintura y cadera fueron medidos con una cinta métrica flexible y en ayuno, siempre la paciente en bipedestación, midiendo a nivel del ombligo 
Tabla 2: Criterios de severidad de colecistitis aguda según las guías Tokio 18.

\section{Grado III (severa)}

Colecistitis aguda asociada a disfunción de alguno de los siguientes órganos/sistemas:

1. Disfunción cardiovascular: hipotensión que requiere tratamiento con dopamina $\geq 5 \mu \mathrm{g} / \mathrm{kg}$ por minuto o epinefrina a cualquier dosis

2. Disfunción neurológica: alteración de la conciencia

3. Disfunción respiratoria: $\mathrm{PaO} 2 / \mathrm{FiO} 2$ ratio $<300$

4. Disfunción renal: oliguria, creatinina $>2.0 \mathrm{mg} / \mathrm{dl}$

5. Disfunción hepática: PT-INR $>1.5$

6. Disfunción hematológica: conteo plaquetario $<100,000 / \mathrm{mm} 3$

\section{Grado II (moderada)}

Colecistitis aguda asociada a alguna de las siguientes condiciones:

1. Leucocitosis $>18,000 / \mathrm{mm} 3$

2. Masa palpable en cuadrante superior derecho

3. Duración de los síntomas $>72 \mathrm{~h}$

4. Inflamación local marcada (gangrena, absceso perivesicular o hepático, enfisema, empiema, mucocele, etc.)

\section{Grado I (leve)}

Colecistitis aguda que no cumple criterios para grado III o II. También se puede definir como una colecistitis aguda en un paciente previamente sano, sin disfunción orgánica o cambios inflamatorios moderados en la vesícula, y cuya colecistectomía se realiza de manera segura y con bajo riesgo operatorio

$\mathrm{PT}=$ tiempo de protrombina, INR = International Normalized Ration para la cintura y a nivel de la parte glútea más prominente para la cadera.

Al no realizarse ninguna intervención experimental, este estudio no requirió de aprobación por el comité de bioética institucional, si se cuenta con el consentimiento informado de los pacientes.

Se hizo un análisis estadístico de las variables con el programa SPSS (versión 23.0; Chicago, II), las variables cualitativas se compararon con la prueba de $\chi^{2}, y$ las variables cuantitativas con la prueba $t$ de Student (variables de comportamiento normal) y la prueba $U$ de Mann-Whitney (variables de comportamiento anormal). Se consideró el valor de p como significativo si éste era $<0.05$.

\section{RESULTADOS}

Se evaluaron 132 pacientes que cumplían con los criterios de inclusión, con un promedio de edad de 38.6 años, un promedio de IMC 31.8 $\mathrm{kg} / \mathrm{m}^{2}$, un promedio de triglicéridos de 164 $\mathrm{mg} / \mathrm{dl}$, el resto de los valores se muestran en la Tabla 3.

Tabla 3: Características numéricas de total de pacientes estudiados.

\begin{tabular}{lccc} 
Variable & Media \pm DE & Mínimo & Máximo \\
\hline Edad (años) & $38.6 \pm 10.6$ & 20 & 69 \\
Peso (kg) & $77.4 \pm 15.4$ & 45 & 115 \\
Talla (cm) & $156.0 \pm 6.2$ & 140 & 173 \\
IMC $\left(\mathrm{kg} / \mathrm{m}^{2}\right)$ & $31.8 \pm 6.1$ & 18.7 & 48.5 \\
Cintura (cm) & $101.1 \pm 13.7$ & 67 & 130 \\
Cadera (cm) & $110.7 \pm 12.3$ & 80 & 145 \\
ICC & $0.9 \pm 0.1$ & 0.72 & 1.09 \\
Tensión sistólica (mmHg) & $118.6 \pm 17.2$ & 90 & 180 \\
Tensión diastólica (mmHg) & $74.7 \pm 11.8$ & 50 & 110 \\
Glucosa (mg/dl) & $104.2 \pm 32.6$ & 70 & 344 \\
Colesterol (mg/dl) & $186.8 \pm 48.4$ & 84 & 407 \\
HDL (mg/dl) & $40.7 \pm 9.8$ & 22 & 67 \\
Triglicéridos (mg/dl) & $164.1 \pm 75.5$ & 47 & 505 \\
Leucocitos ( $\left.\times 10^{9} / \mathrm{l}\right)$ & $10.4 \pm 10.1$ & 4 & 18 \\
Neutrófilos (\%) & $67.3 \pm 13.6$ & 39 & 97 \\
Tiempo quirúrgico (minutos) & $64.5 \pm 27.3$ & 30 & 200 \\
\hline
\end{tabular}

$\mathrm{DE}$ = desviación estándar; IMC = índice de masa corporal; $\mathrm{ICC}=$ índice cintura/cadera; HDL = lipoproteínas de alta densidad.

Fuente: archivo del HGZ No. 35 - IMSS. 


\begin{tabular}{|c|c|c|}
\hline \multirow[b]{2}{*}{ Variable } & \multicolumn{2}{|c|}{$\begin{array}{l}\text { Total de pacientes } \\
\qquad(\mathrm{N}=132)\end{array}$} \\
\hline & n & $\%$ \\
\hline \multicolumn{3}{|l|}{ DM2 } \\
\hline No & 80 & 60.6 \\
\hline Sí & 52 & 39.4 \\
\hline \multicolumn{3}{|l|}{ HTA } \\
\hline No & 120 & 90.9 \\
\hline Sí & 12 & 9.1 \\
\hline \multicolumn{3}{|l|}{ Hipertrigliceridemia } \\
\hline No & 64 & 48.5 \\
\hline Sí & 68 & 51.5 \\
\hline \multicolumn{3}{|l|}{ Hipercolesterolemia } \\
\hline No & 89 & 67.4 \\
\hline Sí & 43 & 32.6 \\
\hline \multicolumn{3}{|l|}{ Dislipidemia } \\
\hline No & 52 & 39.4 \\
\hline Sí & 80 & 60.6 \\
\hline \multicolumn{3}{|l|}{ IMC } \\
\hline Normopeso & 18 & 13.6 \\
\hline Sobrepeso & 32 & 24.2 \\
\hline Obesidad & 82 & 62.1 \\
\hline \multicolumn{3}{|l|}{ Obesidad abdominal } \\
\hline No & 23 & 17.4 \\
\hline Sí & 109 & 82.6 \\
\hline \multicolumn{3}{|l|}{ Obesidad ICC } \\
\hline No & 23 & 17.4 \\
\hline Sí & 109 & 82.6 \\
\hline \multicolumn{3}{|l|}{ SM } \\
\hline No & 59 & 44.7 \\
\hline Sí & 73 & 55.3 \\
\hline \multicolumn{3}{|c|}{$\begin{array}{l}\text { DM2 = diabetes mellitus tipo } 2 \text {; HTA = hipertensión } \\
\text { arterial; IMC = índice de masa corporal; ICC = índi- } \\
\text { ce cintura/cadera; SM = síndrome metabólico. } \\
\text { Fuente: archivo del HGZ No. } 35 \text { - IMSS. }\end{array}$} \\
\hline
\end{tabular}

En lo que respecta a las comorbilidades presentadas en el total de pacientes, se encontró una prevalencia de DM2 de $39.4 \%$, de HTA de $9.1 \%$, hipertrigliceridemia de $51.5 \%$, entre otros. El resto de los porcentajes se describen en la Tabla 4.

Se compararon las variables cuantitativas por grupos (grado I vs. grado II) y se observaron diferencias estadísticamente significativas en el promedio de triglicéridos, leucocitos y neutrófilos, el resto de los promedios que no fueron significativos se muestran en la Tabla 5.

En lo que respecta a las comorbilidades entre los grupos, se apreció una diferencia estadísticamente significativa en la presencia de hipertrigliceridemia y dislipidemia, el resto de las comorbilidades que no fueron significativas se detallan en la Tabla 6.

\section{DISCUSIÓN}

La colecistitis aguda es la complicación más común de la colelitiasis, con una mortalidad aproximada de 3\%. El tratamiento estándar es la colecistectomía laparoscópica; ${ }^{6}$ sin embargo, la colecistectomía abierta continúa vigente en algunos centros de segundo nivel de atención.?

El sobrepeso y la obesidad incrementan el riesgo de colelitiasis ${ }^{8}$ mediante la mayor secreción de colesterol en hígado, lo que produce la sobresaturación y precipitación de la bilis con la consecuente formación de cálculos. Está claro el papel de la obesidad en la génesis de la litiasis vesicular, pero su papel es discutible con respecto a la severidad. La presencia de obesidad en el contexto de colelitiasis supone un estado inflamatorio visceral de tipo crónico que podría influir en la severidad de un cuadro de colecistitis aguda; sin embargo, en este estudio los promedios entre los grupos (grado I vs. grado II) no fueron significativos en relación con el IMC, cintura, cadera e ICC. Esto podría sugerir que, aunque la condición de obesidad predispone a un estado inflamatorio visceral al inicio de la enfermedad, cuando la colecistitis esta instaurada, la obesidad per se parece no influir en la gravedad del cuadro.

Aunque no está demostrada la relación entre los niveles séricos de colesterol total y la frecuencia de colelitiasis, pudimos observar que tanto el promedio de triglicéridos (155.9 vs. $178.4 ; \mathrm{p}=0.008)$ como la presencia de hipertrigliceridemia $(40.5 \%$ vs. $70.8 \% ; p=$ $0.001)$ ) sí se correlacionaron con el grupo 2 (colecistitis grado II). Las alteraciones en el metabolismo de los lípidos son un elemento pivote en el desarrollo de la colelitiasis en los pacientes con obesidad. Esta dualidad se caracteriza por hipertrigliceridemia que se correlaciona con 


\section{Tabla 5: Características numéricas de los grupos estudiados.}

\begin{tabular}{|c|c|c|c|}
\hline Variable & $\begin{array}{c}\text { Grupo } 1(n=84) \\
\text { Media } \pm \text { DE }\end{array}$ & $\begin{array}{c}\text { Grupo } 2(n=48) \\
\text { Media } \pm \text { DE }\end{array}$ & $\mathbf{p}$ \\
\hline Edad (años) & $39.1 \pm 10.3$ & $37.7 \pm 11.0$ & $0.48 *$ \\
\hline Peso (kg) & $77.2 \pm 16.6$ & $77.6 \pm 13.1$ & $0.88 *$ \\
\hline Talla $(\mathrm{cm})$ & $156.3 \pm 6.3$ & $155.4 \pm 6.2$ & $0.42 *$ \\
\hline $\mathrm{IMC}\left(\mathrm{kg} / \mathrm{m}^{2}\right)$ & $31.6 \pm 6.6$ & $32.1 \pm 5.2$ & $0.61 *$ \\
\hline Cintura $(\mathrm{cm})$ & $100 \pm 13.7$ & $103.1 \pm 13.7$ & $0.21 *$ \\
\hline Cadera $(\mathrm{cm})$ & $110.1 \pm 13.0$ & $111.7 \pm 11.0$ & $0.48 *$ \\
\hline $\mathrm{ICC}$ & $0.9 \pm 0.1$ & $0.9 \pm 0.1$ & $0.23 *$ \\
\hline Tensión sistólica (mmHg) & $118.8 \pm 17.8$ & $118.3 \pm 16.1$ & $0.87 *$ \\
\hline Tensión diastólica (mmHg) & $75.1 \pm 11.9$ & $74 \pm 11.7$ & $0.61 *$ \\
\hline Glucosa (mg/dl) & $101.8 \pm 24.6$ & $108.3 \pm 43.1$ & $0.26^{*}$ \\
\hline Colesterol (mg/dl) & $183.2 \pm 48.9$ & $193.1 \pm 47.3$ & $0.26^{*}$ \\
\hline $\mathrm{HDL}(\mathrm{mg} / \mathrm{dl})$ & $41.8 \pm 9.8$ & $38.6 \pm 9.7$ & $0.06 * *$ \\
\hline Triglicéridos (mg/dl) & $155.9 \pm 81.6$ & $178.4 \pm 61.6$ & $0.008 * *$ \\
\hline Leucocitos $\left(\times 10^{9} / 1\right)$ & $9.7 \pm 12.2$ & $11.6 \pm 4.3$ & $<0.01 * *$ \\
\hline Neutrófilos $(\%)$ & $63.1 \pm 12.6$ & $74.8 \pm 12.0$ & $<0.01^{*}$ \\
\hline Tiempo quirúrgico (minutos) & $61.4 \pm 20.2$ & $69.9 \pm 36.1$ & $0.14 *$ \\
\hline \multicolumn{4}{|c|}{$\begin{array}{l}\text { * t de Student; ** U de Mann-Whitney. } \\
\mathrm{DE}=\text { desviación estándar; IMC = índice de masa corporal; ICC = índice cintura/cadera; HDL = lipoproteínas de alta } \\
\text { densidad. } \\
\text { Fuente: Archivo del HGZ No. 35, IMSS. }\end{array}$} \\
\hline
\end{tabular}

falta de motilidad de la vesícula biliar ${ }^{9}$ y por lo tanto, un factor en la génesis de litiasis. Los resultados de este estudio sugieren que los niveles elevados de triglicéridos sí aumentan el riesgo de severidad (al menos al grado II) de manera significativa, lo que condicionaría tanto la aparición de una agudización del cuadro como un factor de riesgo de severidad.

Otros estudios han sugerido que la obesidad puede ser un factor protector contra la severidad en la colelitiasis en pacientes masculinos, refiriendo que la grasa visceral puede tener una función protectora contra el estado inflamatorio en la colecistitis. ${ }^{8}$ Estos mismos resultados no se han reproducido en otros estudios. ${ }^{10,11} \mathrm{La}$ colelitiasis tiene una relación directa con el hígado grado no alcohólico, ${ }^{12}$ y la infiltración grasa en los órganos viscerales como el hígado puede provocar inflamación crónica, por lo que suponer que la grasa visceral condiciona protección podría ser contradictorio. ${ }^{13}$

La DM2 y la HTA se consideran factores de riesgo de desarrollar colecistitis aguda. ${ }^{14}$
Fagan y colaboradores demostraron que la DM2 sí influyó en el desarrollo de colecistitis gangrenosa; ${ }^{15}$ sin embargo, nuestros resultados sugieren que tanto la DM2 como la HTA parecen no influir en el desarrollo de complicaciones, lo anterior está sujeto a debate y se requieren estudios más amplios para corroborar el verdadero papel que desempeñan estas comorbilidades en la severidad de la colecistitis.

\section{CONCLUSIÓN}

En el presente estudio, los resultados sugieren qué niveles elevados de triglicéridos podrían actuar como factor de riesgo de padecer colecistitis grado II. No se demostró relación entre la obesidad medida por el IMC, cintura, cadera y/o ICC con el grado de severidad de la colecistitis (grado I vs. grado II). Sin embargo, hacen falta más estudios de tipo prospectivo o estudios multicéntricos para corroborar estos hallazgos. 
Tabla 6: Comorbilidades presentes en los pacientes.

\begin{tabular}{|c|c|c|c|c|c|}
\hline \multirow[b]{2}{*}{ Variable } & \multicolumn{2}{|c|}{$\begin{array}{c}\text { Grupo } 1 \\
(\mathrm{n}=84)\end{array}$} & \multicolumn{2}{|c|}{$\begin{array}{c}\text { Grupo } 2 \\
(n=48)\end{array}$} & \multirow[b]{2}{*}{ p* } \\
\hline & $\mathbf{n}$ & $\%$ & $\mathbf{n}$ & $\%$ & \\
\hline \multicolumn{6}{|l|}{ DM2 } \\
\hline No & 50 & 59.5 & 30 & 62.5 & 0.73 \\
\hline Sí & 34 & 40.5 & 18 & 37.5 & \\
\hline \multicolumn{6}{|l|}{ HTA } \\
\hline No & 77 & 91.7 & 43 & 89.6 & 0.68 \\
\hline Sí & 7 & 8.3 & 5 & 10.4 & \\
\hline \multicolumn{6}{|l|}{ Hipertrigliceridemia } \\
\hline No & 50 & 59.5 & 14 & 29.2 & 0.001 \\
\hline Sí & 34 & 40.5 & 34 & 70.8 & \\
\hline \multicolumn{6}{|l|}{ Hipercolesterolemia } \\
\hline No & 57 & 67.9 & 32 & 66.7 & 0.88 \\
\hline Sí & 27 & 32.1 & 16 & 33.3 & \\
\hline \multicolumn{6}{|l|}{$\mathrm{HDL}(<50 \mathrm{mg} / \mathrm{dl})$} \\
\hline No & 19 & 22.6 & 7 & 14.6 & 0.26 \\
\hline Sí & 65 & 77.4 & 41 & 85.4 & \\
\hline \multicolumn{6}{|l|}{ Dislipidemia } \\
\hline No & 41 & 48.8 & 11 & 22.9 & 0.003 \\
\hline Sí & 43 & 51.2 & 37 & 77.1 & \\
\hline \multicolumn{6}{|l|}{ IMC } \\
\hline Normopeso & 14 & 16.7 & 4 & 8.3 & 0.24 \\
\hline Sobrepeso & 22 & 26.2 & 10 & 20.8 & \\
\hline Obesidad & 48 & 57.1 & 34 & 70.8 & \\
\hline \multicolumn{6}{|l|}{ IMC } \\
\hline$<30$ & 34 & 40.5 & 14 & 29.2 & 0.19 \\
\hline$\geq 30$ & 50 & 59.5 & 34 & 70.8 & \\
\hline \multicolumn{6}{|l|}{ Obesidad abdominal } \\
\hline No & 15 & 17.9 & 8 & 16.7 & 0.86 \\
\hline Sí & 69 & 82.1 & 40 & 83.3 & \\
\hline \multicolumn{6}{|l|}{ Obesidad por ICC } \\
\hline No & 17 & 20.2 & 6 & 12.5 & 0.26 \\
\hline Sí & 67 & 79.8 & 42 & 87.5 & \\
\hline \multicolumn{6}{|l|}{ SM } \\
\hline No & 42 & 50.0 & 17 & 35.4 & 0.1 \\
\hline Sí & 42 & 50.0 & 31 & 64.6 & \\
\hline
\end{tabular}

${ }^{*} \chi^{2}$.

DM2 = diabetes mellitus tipo 2; HTA = hipertensión arterial; $\mathrm{HDL}=$ lipoproteínas de alta densidad; IMC = índice de masa corporal; ICC = índice cintura/cadera; $\mathrm{SM}=$ síndrome metabólico.

Fuente: Archivo del HGZ No. 35, IMSS. 


\section{REFERENCIAS}

1. Stinton LM, Shaffer EA. Epidemiology of gallbladder disease: cholelithiasis and cancer. Gut Liver. 2012; 6: 172-187.

2. Gallagher TK, Parks RW. Gallstones. Surg. 2014; 32: 635-642.

3. Díaz-Rosales JD, Enríquez-Domínguez L, AlcocerMoreno JA, Romo JE, Duarte E, Díaz-Torres B. Association of central obesity and severity in cholelithiasis during cholecystectomy in adult women. World J Med Med Sci Res. 2015; 3: 4-6.

4. Yokoe M, Hata J, Takada T, et al. Tokyo Guidelines 2018: diagnostic criteria and severity grading of acute cholecystitis (with videos). J Hepatobiliary Pancreat Sci. 2018; 25: 41-54.

5. Sanford DE. An update on technical aspects of cholecystectomy. Surg Clin North Am. 2019; 99: 245258.

6. Lee SO, Yim SK. Management of acute cholecystitis. Korean J Gastroenterol. 2018; 71: 264-268.

7. García-Chávez J, Ramírez-Amezcua FJ. Colecistectomía de urgencia laparoscópica versus abierta. Cir Gen. 2012; 34: 174-178.

8. Chauhan VV, Shah BA, Mahadik SJ, Videkar RP. Evaluation of relationship of body mass index with severity of cholecystitis. Int Surg J. 2019; 6: 868-875.

9. Méndez-Sánchez N, Chávez-Tapia NC, Uribe M. Obesidad y litiasis. Gac Med Mex. 2004; 140: S59-S66.

10. Dubhashi SP, Trinath T. Is severity of cholecystitis related to body mass index? J Int Med Sci Acad. 2013; 26: 101-102.

11. Lee HK, Han HS, Min SK. The association between body mass index and the severity of cholecystitis. Am J Surg. 2009; 197: 455-458.

12. Díaz-Rosales JD, Enríquez-Domínguez L, Díaz-Torres B. Factores de riesgo para hígado graso no alcohólico en pacientes con colelitiasis sintomática. Arch Med. 2016; 16: 98-108.
13. Díaz-Rosales JD, Alcocer-Moreno JA, EnríquezDomínguez L. Síndrome metabólico y colecistitis complicada en mujeres adultas. Arch Med. 2016; 16 : 304-311.

14. Lee S, Chung CW, Ko KH, Kwon SW. Risk factors for the clinical course of cholecystitis in patients who undergo cholecystectomy. Korean J Hepatobiliary Pancreat Surg. 2011; 15: 164-170.

15. Fagan SP, Awad SS, Rahwan K, et al. Prognostic factors for the development of gangrenous cholecystitis. Am J Surg. 2003; 186: 481-485.

Consideraciones y responsabilidad ética: Privacidad de los datos. De acuerdo a los protocolos establecidos en el centro de trabajo de los autores, éstos declaran que han seguido los protocolos sobre la privacidad de datos de pacientes preservando su anonimato. El consentimiento informado del paciente referido en el artículo se encuentra en poder del autor.

Financiamiento: No se recibió apoyo financiero para la realización de este trabajo.

Conflicto de intereses: Los autores declaran que no existe ningún conflicto de intereses en la realización del trabajo.

Correspondencia:

Juan de Dios Díaz-Rosales

Servicio de Endoscopia Gastrointestinal,

Hospital General de Zona No. 35, IMSS.

Av. Valentín Fuentes Núm. 2582,

Col. Infonavit Casas Grandes, 32600,

Ciudad Juárez, Chihuahua.

Tel: (656) 3751759

E-mail: jdedios.diaz@uacj.mx 